\title{
Uncertainty relations for time-averaged weak values
}

\author{
Eli Pollak ${ }^{1, *}$ and Salvador Miret-Artés ${ }^{2, \dagger}$ \\ ${ }^{1}$ Chemical and Biological Physics Department, Weizmann Institute of Science, 76100 Rehovoth, Israel \\ ${ }^{2}$ Instituto de Física Fundamental, Consejo Superior de Investigaciones Científicas, Serrano 123, 28006 Madrid, Spain
}

(Received 29 August 2018; published 8 January 2019)

\begin{abstract}
Time averaging of weak values using the quantum transition path time probability distribution enables us to establish a general uncertainty relation for the weak values of two not necessarily Hermitian operators. This new relation is a weak value analog of the Schrödinger strong value uncertainty relation. It leads to the conclusion that it is possible to determine with high accuracy the simultaneous mean weak values of noncommuting operators by judicious choice of the pre- and postselected states even when the postselected state is not an eigenfunction of one of the respective operators. When the time fluctuations of the two weak values are proportional to each other there is no uncertainty limitation on their variances and, in principle, their means can be determined with arbitrary precision even though their corresponding operators do not commute. To exemplify these properties we consider specific weak value uncertainty relations for the time-energy, coordinate-momentum, and coordinatekinetic-energy pairs. In addition we analyze spin operators and the Stern-Gerlach experiment in weak and strong inhomogeneous magnetic fields. This classic case leads to anomalous spin values when the field is weak. The weak value uncertainty relation implies that anomalous spin values are associated with large variances so that their measurement demands increased signal averaging. These examples establish the importance of considering the time dependence of weak values in scattering experiments.
\end{abstract}

DOI: 10.1103/PhysRevA.99.012108

\section{INTRODUCTION}

The uncertainty principle is a fundament of quantum mechanics. The "intrinsic" uncertainty principle was derived by Robertson [1] in 1929. He showed that the product of the standard deviations of the diagonal elements of two noncommuting Hermitian operators is bounded from below by the diagonal element of their commutator. For the coordinate momentum pair this lower bound is $\hbar / 2$. Schrödinger further tightened the bound by including the anticommutator [2]. Heisenberg, in his consideration of an uncertainty principle, seems to have had something else in mind, namely a measurement disturbance relation whereby measurement of one property of a system, say its position, creates an uncertainty in its momentum. It was only 15 years ago that the fundamental measurement disturbance relation was derived by Ozawa [3], who also showed that this relation is not necessarily bounded from below by the Robertson-Schrödinger lower bound.

The pioneering work by Aharonov et al. [4], which showed among others that the results of weak measurement may be described by weak values of operators, has attracted the attention of many theorists [5-7] and experimentalists [8-11]. This led also to the consideration of the measurement disturbance relation in the context of weak values by Lund and Weisman [12]. They showed how Ozawa's result could be measured experimentally by considering weak measurements and weak values. Their protocol was then implemented experimentally by Steinberg and co-workers [13].

\footnotetext{
*eli.pollak@weizmann.ac.il

${ }^{\dagger}$ s.miret@iff.csic.es
}

The uncertainty relation developed in this paper is somewhat different. It is the application of the Schrödinger type of intrinsic uncertainty relation within the context of weak values and time evolution. The model underlying our considerations is the typical weak measurement experiment. It is a scattering experiment which for simplicity occurs in one dimension. For example, a particle is scattered by a potential. It originates at the initial time to the left of the potential, interacts with it, and is then either transmitted to its right where it continues motion as a free particle or reflected likewise to the left. A "screen" is set up to the right of the potential and the momentum of the particle is weakly measured prior but arbitrarily close to the location of the screen. In this way the momentum weak value is measured at the postselected location of the screen. However, and this is critical, the particle, if it is transmitted, will arrive at the screen at varying time and therefore with varying values of the weak momentum. This creates a distribution in time of momentum weak values. There is no way in which one can know in advance at what time the particle will reach the weak measurement device and it will typically reach the weak measurement device at different times so that the result of the measurement may vary. It is therefore necessary to also study the statistics of the associated time-dependent weak value distribution and this leads us to a weak value Schrödinger type uncertainty relation. It is intrinsic yet at the same time intimately related to the weak value.

The probability distribution which determines the time statistics is the so-called quantum transition path time probability distribution [14]. In Ref. [15] we showed how such time averaging of weak values leads to a well-defined timeenergy uncertainty principle and a time-energy weak value commutation relation. We also showed how with suitable 
postselection it is possible to predict in advance the strong momentum value of a single particle at a postselected position with an accuracy greater than the Robertson limit of $\hbar / 2$. In these studies time is considered to be an external parameter as discussed by Aharonov and Bohm [16] and Busch [17].

The topic of this paper is to consider the general scenario of the time statistics of weak values to derive a Schrödinger-like uncertainty relation for the time-averaged standard deviation of noncommuting, not necessarily Hermitian operators. To implement this goal we develop in Sec. II an algebra of what we define to be postselected operators, their commutation relations, and time averages. This is then used to derive a weak value uncertainty relation for the product of the standard deviations of the postselected operators in terms of their timeaveraged commutators and anticommutators.

We then consider in Sec. III some specific cases which exemplify the utility of the weak value uncertainty relation. The first example is the rederivation of the time-energy uncertainty relation for weak values. We then consider timedependent weak values for postselected (normalized) coherent states. Due to this choice the time-averaged weak values of the coordinate and momentum are found to be linearly proportional to each other. This implies through the weak value uncertainty relation that by judicious choice of the pre- and postselected states both quantities can be determined with arbitrary precision even though the postselected state is not an eigenstate of one of the relevant operators. We then show how any uncertainty in the time-averaged weak value of the position coordinate leads to an uncertainty in the time-averaged weak value of the kinetic energy. Through the time-energy uncertainty relation this means that increasing the accuracy in the determination of the position can lead to an increased indeterminacy in the time at which the weak value can be measured. As a fourth example we choose the paradigmatic Stern-Gerlach experiment which is analyzed in terms of time-dependent spin weak values in the presence of weak and strong inhomogeneous magnetic fields. Here too the fluctuations of the weak values of the spin about their mean display a proportionality so that the uncertainty principle does not impose any restriction on the time-averaged spin weak values even though the corresponding operators do not commute and the pre- and postselected states are not eigenstates of the spin operators. Interestingly, the weak value uncertainty relation leads to the conclusion that the magnitude of the standard deviation of the spin weak value is proportional to the magnitude of the time weighted mean spin weak value. This means that anomalously large spin weak values lead to a large uncertainty, implying, from an experimental point of view, that the weak values have to be measured many times in order to obtain a reliable estimate of the mean value. This sheds light on recent neutron interferometry experiments [18,19], where it was found that strong interaction measurement outperforms weak interaction when it comes to precision and accuracy of the measurement.

To further elucidate the implications of the weak value uncertainty relation we consider in Sec. IV two specific models. The first is one-dimensional potential scattering to exemplify the coordinate, momentum, energy, and time uncertainties. The second is a numerical analysis of the Stern-Gerlach setup in the presence of weak and strong inhomogeneous magnetic fields. Perhaps the most striking result here is that anomalous weak values are associated with large standard deviations as compared to the "regular" ones. Finally, in Sec. V, we further discuss the implications of the weak value uncertainty relation and the importance of taking into consideration the time evolution of weak values.

\section{TIME AVERAGING AND A WEAK VALUE UNCERTAINTY PRINCIPLE}

\section{A. Weak value operator algebra}

In this section we formulate and develop an operator formalism for weak values. We assume a postselected normalized state $|\Phi\rangle$ and define the density operator associated with it as

$$
\hat{D}_{\Phi}=|\Phi\rangle\langle\Phi| .
$$

The density operator is by definition Hermitian and it is a projection operator, that is $\hat{D}_{\Phi}^{2}=\hat{D}_{\Phi}$. The weak value operator $\hat{O}_{\Phi}$ associated with the operator $\hat{O}$ and the postselected state is defined as the product

$$
\hat{O}_{\Phi}=\hat{D}_{\Phi} \hat{O}
$$

With this definition the weak value of the operator $\hat{O}$ associated with the preselected (normalized) state $|\Psi\rangle$ and postselected state $|\Phi\rangle$ is given by a ratio of strong values [20] with the preselected state:

$$
O_{w}(\Phi ; \Psi)=\frac{\langle\Phi|\hat{O}| \Psi\rangle}{\langle\Phi \mid \Psi\rangle}=\frac{\left\langle\Psi\left|\hat{O}_{\Phi}\right| \Psi\right\rangle}{\left\langle\Psi\left|\hat{D}_{\Phi}\right| \Psi\right\rangle}
$$

A central goal of this paper is to present time averaging of weak values with corresponding general uncertainty relations. For the chosen pre- and postselected states the time probability distribution is defined to be [14]

$$
P_{\Psi}(t ; \Phi)=\frac{\left\langle\Psi_{t}\left|\hat{D}_{\Phi}\right| \Psi_{t}\right\rangle}{\int_{0}^{\infty} d t\left\langle\Psi_{t}\left|\hat{D}_{\Phi}\right| \Psi_{t}\right\rangle} \equiv \frac{\left\langle\Psi_{t}\left|\hat{D}_{\Phi}\right| \Psi_{t}\right\rangle}{N(\Phi ; \Psi)},
$$

where $\left|\Psi_{t}\right\rangle$ is the time evolved preselected state under the Hamiltonian $\hat{H}$ :

$$
\left|\Psi_{t}\right\rangle=\exp \left(-\frac{i \hat{H} t}{\hbar}\right)|\Psi\rangle .
$$

The time-averaged weak value is then calculated as

$$
\begin{aligned}
\left\langle O_{w}(\Phi ; \Psi)\right\rangle & \equiv \int_{0}^{\infty} d t P_{\Psi}(t ; \Phi) O_{w}\left(\Phi ; \Psi_{t}\right) \\
& =\frac{1}{N(\Phi ; \Psi)} \int_{0}^{\infty} d t\left\langle\Psi_{t}\left|\hat{O}_{\Phi}\right| \Psi_{t}\right\rangle
\end{aligned}
$$

thus relating time-averaged weak values to time integrals of diagonal matrix elements of weak valued operators. It is important to note that if the postselected state $|\Phi\rangle$ is chosen to be an eigenstate of the operator $\hat{O}$ with eigenvalue $o$ then the time-averaged value of the operator is just the eigenvalue $o$.

We then consider two not necessarily Hermitian operators $\hat{A}$ and $\hat{B}$ such that their commutation relation is the antiHermitian operator

$$
\left[\hat{B}^{\dagger}, \hat{A}\right] \equiv \hat{B}^{\dagger} \hat{A}-\hat{A}^{\dagger} \hat{B}
$$


and their anticommutation relation is the Hermitian operator

$$
\left\{\hat{B}^{\dagger}, \hat{A}\right\} \equiv \hat{B}^{\dagger} \hat{A}+\hat{A}^{\dagger} \hat{B}
$$

The commutation and anticommutation relations for the associated weak value operators are then

$$
\begin{aligned}
& {\left[\hat{B}_{\Phi}^{\dagger}, \hat{A}_{\Phi}\right] \equiv \hat{B}^{\dagger} \hat{D}_{\Phi} \hat{A}-\hat{A}^{\dagger} \hat{D}_{\Phi} \hat{B},} \\
& \left\{\hat{B}_{\Phi}^{\dagger}, \hat{A}_{\Phi}\right\} \equiv \hat{B}^{\dagger} \hat{D}_{\Phi} \hat{A}+\hat{A}^{\dagger} \hat{D}_{\Phi} \hat{B},
\end{aligned}
$$

and one notes that they are not the same as the original relations.

As a specific example we choose the coordinate $\hat{q}$ and momentum $\hat{p}$ operators for which the commutator is $i \hbar$ so that the off-diagonal coordinate matrix element of the commutator is

$$
\left\langle x|[\hat{q}, \hat{p}]| x^{\prime}\right\rangle=i \hbar \delta\left(x-x^{\prime}\right) .
$$

The off-diagonal coordinate matrix element for the weak value commutator is quite different:

$$
\left\langle x\left|\left[\hat{q}_{\Phi}^{\dagger}, \hat{p}_{\Phi}\right]\right| x^{\prime}\right\rangle=i \hbar\left[x\langle x \mid \Phi\rangle \frac{\partial\left\langle\Phi \mid x^{\prime}\right\rangle}{\partial x^{\prime}}+\frac{\partial\langle x \mid \Phi\rangle}{\partial x} x^{\prime}\left\langle\Phi \mid x^{\prime}\right\rangle\right] .
$$

This has implications when considering as below the weak value uncertainty relations.

\section{B. Weak value uncertainty relations}

The deviation of the postselected operator $\hat{O}_{\Phi}$ from its time-averaged mean is denoted as

$$
\Delta \hat{O}_{\Phi}=\hat{O}_{\Phi}-|\Phi\rangle\langle\Phi|\left\langle O_{w}(\Phi ; \Psi)\right\rangle .
$$

As before, from the definition of the weak value operator, if the postselected state $|\Phi\rangle$ is an eigenstate of the operator $\hat{O}$ then the deviation $\Delta \hat{O}_{\Phi}$ vanishes. If it is not an eigenstate, then following the Schrödinger derivation of the uncertainty relation $[1,2,21]$ one constructs the following inequality for two not necessarily Hermitian operators $\hat{A}$ and $\hat{B}$ :

$$
\begin{aligned}
0 \leqslant & \frac{1}{N(\Phi ; \Psi)} \int_{0}^{\infty} d t\left\langle\Psi_{t}\right|\left[\Delta \hat{A}_{\Phi}^{\dagger}+i \lambda^{*} \Delta \hat{B}_{\Phi}^{\dagger}\right] \\
& \times\left[\Delta \hat{A}_{\Phi}-i \lambda \Delta \hat{B}_{\Phi}\right]\left|\Psi_{t}\right\rangle,
\end{aligned}
$$

where $\lambda$ is an arbitrary complex number

$$
\lambda=\lambda_{R}+i \lambda_{I}
$$

As we shall see below when analyzing some specific examples, it may happen that even if the two operators $\hat{A}$ and $\hat{B}$ do not commute their weak values are proportional to each other, that is, $\left\langle\Phi\left|\Delta \hat{A}_{\Phi}\right| \Psi_{t}\right\rangle=C\left\langle\Phi\left|\Delta \hat{B}_{\Phi}\right| \Psi_{t}\right\rangle$ with $C$ a proportionality constant. In such a case, the value $i \lambda=C$ causes the right-hand side (RHS) of Eq. (14) to equal zero and there is no constraint on the variances so both may vanish even though the pre- and postselected states are not eigenstates of the operators under consideration. In other words, timeaveraged weak values of noncommuting operators may at times be obtained with certainty.

In the general case, when such a linear relation does not occur, minimizing the right-hand side of the inequality in Eq. (14) with respect to the two time-independent real numbers $\lambda_{R}$ and $\lambda_{I}$ leads to the time-averaged weak value uncertainty relation:

$$
\begin{gathered}
\frac{\int_{0}^{\infty} d t\left\langle\Psi_{t}\left|\Delta \hat{A}_{\Phi}^{\dagger} \Delta \hat{A}_{\Phi}\right| \Psi_{t}\right\rangle}{N(\Phi ; \Psi)} \frac{\int_{0}^{\infty} d t\left\langle\Psi_{t}\left|\Delta \hat{B}_{\Phi}^{\dagger} \Delta \hat{B}_{\Phi}\right| \Psi_{t}\right\rangle}{N(\Phi ; \Psi)} \\
\geqslant \frac{1}{4}\left(\left[\frac{\int_{0}^{\infty} d t\left\langle\Psi_{t}\left|\left\{\Delta \hat{B}_{\Phi}^{\dagger}, \Delta \hat{A}_{\Phi}\right\}\right| \Psi_{t}\right\rangle}{N(\Phi ; \Psi)}\right]^{2}\right. \\
\left.-\left[\frac{\int_{0}^{\infty} d t\left\langle\Psi_{t}\left|\left[\Delta \hat{B}_{\Phi}^{\dagger}, \Delta \hat{A}_{\Phi}\right]\right| \Psi_{t}\right\rangle}{N(\Phi ; \Psi)}\right]^{2}\right)
\end{gathered}
$$

which is the central formal result of this paper. It expresses the minimal standard deviation of the time average of the weak values of two operators whose weak value commutators and anticommutators do not vanish. This weak value uncertainty principle is a direct analog of the strong value uncertainty principle. However, due to the postselection, in practice, it differs from it significantly. As detailed in the Appendix, this formal result may be rewritten also as

$$
\begin{gathered}
\left\langle\left|\Delta A_{w}\left(\Phi ; \Psi_{t}\right)\right|^{2}\right\rangle\left\langle\left|\Delta B_{w}\left(\Phi ; \Psi_{t}\right)\right|^{2}\right\rangle \\
\geqslant\left|\left\langle\Delta A_{w}\left(\Phi ; \Psi_{t}\right) \Delta B_{w}^{*}\left(\Phi ; \Psi_{t}\right)\right\rangle\right|^{2},
\end{gathered}
$$

where we have used the notation for the time-dependent fluctuation of the weak values:

$$
\Delta A_{w}\left(\Phi ; \Psi_{t}\right)=A_{w}\left(\Phi ; \Psi_{t}\right)-\left\langle A_{w}\left(\Phi ; \Psi_{t}\right)\right\rangle .
$$

This result is somewhat more general than the formal uncertainty expression given in Eq. (16) since it is valid also in the case that the postselected state is not normalizable, for example, it might be the continuum state $|x\rangle$.

From Eq. (17), we again note that if the postselected state is an eigenfunction of either the operator $\hat{A}$ or $\hat{B}$ then there is no uncertainty. An important example is when the postselected state is $|x\rangle$ and the two operators are the coordinate and momentum operators. There is no uncertainty in determining precisely the weak values of the coordinate and momentum simultaneously, that is, at the same elapsed time. This reflects a fundamental difference between strong and weak values. In the case of strong values, if the operators do not commute, the uncertainty relation is never trivial. For weak values, even if the operators do not commute, the measure of uncertainty depends on the choice of the postselected state and may vanish. It is thus possible to know with high accuracy the mean weak values of noncommuting operators by judicious choice of the pre- and postselected states. A specific case, exemplifying this result, has been previously considered [15] where we showed that in a scattering experiment, using a preselected coherent state, one may know after the event is over the simultaneous values of the coordinate and the momentum.

\section{EXAMPLES OF WEAK VALUE UNCERTAINTY RELATIONS}

\section{A. Weak value time-energy uncertainty relation}

The weak value time-energy uncertainty relation discussed in Ref. [15] is considered here in the framework of the general formalism of the previous section. The definition of a time 
operator is even today unsolved, in the sense that there is no Hermitian time operator $\hat{T}$ whose commutation relation with the energy operator $\hat{H}$ is $i \hbar$. Instead of employing a time operator we consider it as a parameter multiplying the identity operator $\hat{I}$. Its weak value for any pre- and postselected states is therefore

$$
t_{w}\left(\Phi ; \Psi_{t}\right)=\frac{\left\langle\Phi|t \hat{I}| \Psi_{t}\right\rangle}{\left\langle\Phi \mid \Psi_{t}\right\rangle}=t
$$

and its time average

$$
\left\langle t_{w}(\Phi ; \Psi)\right\rangle=\int_{0}^{\infty} d t P_{\Psi}(t ; \Phi) t
$$

will depend on the pre- and postselected states. When considering the weak value of the time using the definition of Eq. (19), the weak time value deviation as defined in Eq. (18) will generically not vanish, independent of the choice of the pre- and postselected states.

The energy weak value is by definition

$$
E_{w}\left(\Phi ; \Psi_{t}\right)=\frac{\left\langle\Phi|\hat{H}| \Psi_{t}\right\rangle}{\left\langle\Phi \mid \Psi_{t}\right\rangle}=i \hbar \frac{\partial}{\partial t} \ln \left\langle\Phi \mid \Psi_{t}\right\rangle
$$

and its mean may be written as

$$
\left\langle E_{w}\left(\Phi ; \Psi_{t}\right)\right\rangle=\frac{i \hbar}{N(\Phi ; \Psi)} \int_{0}^{\infty} d t\left\langle\Psi_{t} \mid \Phi\right\rangle \frac{\partial}{\partial t}\left\langle\Phi \mid \Psi_{t}\right\rangle
$$

from which it becomes clear that

$$
\operatorname{Im}\left\langle E_{w}\left(\Phi ; \Psi_{t}\right)\right\rangle=-\frac{\hbar}{2 N(\Phi ; \Psi)}\left|\left\langle\Phi \mid \Psi_{0}\right\rangle\right|^{2} .
$$

If initially the pre- and postselected states do not overlap, the imaginary energy value vanishes. We then find, using an integration by parts, that the time mean of the time-energy weak value commutator is given by

$$
\begin{aligned}
& \left\langle\left[\Delta t_{w}\left(\Phi ; \Psi_{t}\right), \Delta E_{w}\left(\Phi ; \Psi_{t}\right)\right]\right\rangle \\
& =\left\langle\left(t-\left\langle t_{w}(\Phi ; \Psi)\right\rangle\right)\left[\Delta E_{w}^{*}\left(\Phi ; \Psi_{t}\right)-\Delta E_{w}\left(\Phi ; \Psi_{t}\right)\right]\right\rangle \\
& =i \hbar-i \hbar\left\langle t_{w}(\Phi ; \Psi)\right\rangle \frac{\left|\left\langle\Phi \mid \Psi_{0}\right\rangle\right|^{2}}{N(\Phi ; \Psi)} \text {. }
\end{aligned}
$$

In contrast to the difficulty in defining time operators which obey such a commutation relation, we find that when using time averaging of weak values, the energy-time commutation relation emerges naturally. Furthermore, when the initial overlap of the pre- and postselected states vanishes one regains the "standard" ( $i \hbar)$ value for the commutator. It is not possible to obtain such a general result for the anticommutator, for which one needs more knowledge about the specific system and preand postselected states under study.

The result for the commutator then implies the time-energy weak value uncertainty relation

$$
\begin{gathered}
\left\langle\Delta t_{w}(\Phi ; \Psi) \Delta t_{w}^{*}(\Phi ; \Psi)\right\rangle\left\langle\Delta E_{w}(\Phi ; \Psi) \Delta E_{w}^{*}(\Phi ; \Psi)\right\rangle \\
\geqslant \frac{\hbar^{2}}{4}\left(1-\left\langle t_{w}(\Phi ; \Psi)\right\rangle \frac{\left|\left\langle\Phi \mid \Psi_{0}\right\rangle\right|^{2}}{N(\Phi ; \Psi)}\right)^{2}
\end{gathered}
$$

and this reduces to the standard estimate of $\hbar^{2} / 4$ when initially the overlap of the pre- and postselected states vanishes.

\section{B. Coordinate-momentum weak value uncertainty with postselected coherent states}

As a second example we will study the weak values of the coordinate and momentum operators assuming that the postselected state is the coherent state:

$$
\langle x \mid \Phi\rangle=\left(\frac{\Gamma}{\pi}\right)^{1 / 4} \exp \left(-\frac{\Gamma}{2}\left(x-x_{f}\right)^{2}+\frac{i}{\hbar} p_{f}\left(x-x_{f}\right)\right) .
$$

Due to the Gaussian form of the postselected coherent state the weak values of the momentum and the coordinate are linearly related,

$$
p_{w}\left(\Phi ; \Psi_{t}\right)=p_{f}+i \hbar \Gamma\left[x_{f}-x_{w}\left(\Phi ; \Psi_{t}\right)\right]
$$

so that the time induced fluctuations in the weak values of the coordinate and the momentum are proportional to each other:

$$
\Delta p_{w}\left(\Phi ; \Psi_{t}\right)=-i \hbar \Gamma \Delta x_{w}\left(\Phi ; \Psi_{t}\right)
$$

As noted in the previous section, such a linear relation implies that there is no uncertainty limitation on the variances of the time-averaged weak values of the momentum and coordinate and, in principle, they can be known simultaneously with arbitrary precision even though the operators themselves do not commute and the postselected state is not an eigenfunction of either the position or the momentum operators.

\section{Coordinate-kinetic-energy weak value uncertainty with postselected coherent states}

In contrast to the coordinate-momentum pair, when one considers the kinetic-energy-coordinate pair, the corresponding time-averaged weak value uncertainty relation sets a limit on the accuracy with which one may simultaneously determine both weak values. Using the postselected coherent state given by Eq. (26) one readily finds that ( $M$ is the mass of the particle)

$$
\begin{aligned}
T_{w}\left(\Phi ; \Psi_{t}\right)= & \frac{\int_{-\infty}^{\infty} d x\left\langle\Phi\left|p^{2}\right| x\right\rangle\left\langle x \mid \Psi_{t}\right\rangle}{2 M\left\langle\Phi \mid \Psi_{t}\right\rangle} \\
= & \frac{\hbar^{2} \Gamma}{2 M}+\frac{\left(p_{f}+i \hbar \Gamma x_{f}\right)^{2}}{2 M} \\
& -\frac{i \hbar \Gamma\left[p_{f}+i \hbar \Gamma x_{f}\right]}{M} x_{w}\left(\Phi ; \Psi_{t}\right) \\
& -\frac{\hbar^{2} \Gamma^{2}}{2 M} x_{w}^{2}\left(\Phi ; \Psi_{t}\right),
\end{aligned}
$$

so that the fluctuation of the weak value of the kinetic-energy operator is related to the fluctuations of the coordinate and coordinate squared operators as

$$
\begin{aligned}
\Delta T_{w}\left(\Phi ; \Psi_{t}\right)= & -\frac{i \hbar \Gamma\left[p_{f}+i \hbar \Gamma x_{f}\right]}{M} \Delta x_{w}\left(\Phi ; \Psi_{t}\right) \\
& -\frac{\hbar^{2} \Gamma^{2}}{2 M} \Delta x_{w}^{2}\left(\Phi ; \Psi_{t}\right) .
\end{aligned}
$$


With some algebra one then finds that

$$
\left\langle\Delta T_{w}^{*}\left(\Phi ; \Psi_{t}\right) \Delta x_{w}\left(\Phi ; \Psi_{t}\right)\right\rangle=\frac{i \hbar \Gamma\left[p_{f}-i \hbar \Gamma x_{f}\right]}{M}\left\langle\left|\Delta x_{w}\left(\Phi ; \Psi_{t}\right)\right|^{2}\right\rangle-\frac{\hbar^{2} \Gamma^{2}}{2 M}\left\langle\left|\Delta x_{w}\left(\Phi ; \Psi_{t}\right)\right|^{2} \Delta x_{w}^{*}\left(\Phi ; \Psi_{t}\right)\right\rangle
$$

Using the second equality on the RHS of Eq. (17) the weak value uncertainty relation takes the form

$$
\begin{aligned}
\frac{M^{2}}{\hbar^{4} \Gamma^{4}}\left\langle\left|\Delta T_{w}\left(\Phi ; \Psi_{t}\right)\right|^{2}\right\rangle \geqslant & \left(\frac{p_{f}^{2}}{\hbar^{2} \Gamma^{2}}+x_{f}^{2}\right)\left\langle\left|\Delta x_{w}\left(\Phi ; \Psi_{t}\right)\right|^{2}\right\rangle+\frac{1}{4} \frac{\left|\left\langle\left|\Delta x_{w}\left(\Phi ; \Psi_{t}\right)\right|^{2} \Delta x_{w}^{*}\left(\Phi ; \Psi_{t}\right)\right\rangle\right|^{2}}{\left\langle\left|\Delta x_{w}\left(\Phi ; \Psi_{t}\right)\right|^{2}\right\rangle} \\
& +\frac{p_{f}}{\hbar \Gamma}\left\langle\left|\Delta x_{w}\left(\Phi ; \Psi_{t}\right)\right|^{2} \operatorname{Im} \Delta x_{w}\left(\Phi ; \Psi_{t}\right)\right\rangle-x_{f}\left\langle\left|\Delta x_{w}\left(\Phi ; \Psi_{t}\right)\right|^{2} \operatorname{Re} \Delta x_{w}\left(\Phi ; \Psi_{t}\right)\right\rangle,
\end{aligned}
$$

showing that any uncertainty in the time-averaged weak value of the position coordinate leads also to an uncertainty in the kinetic energy. Even if one chooses the postselected state to be localized about $x_{f}=p_{f}=0$ one remains with a nontrivial relation between the two quantities.

\section{Weak values and uncertainty in the Stern-Gerlach experiment}

\section{Model}

The theory of the Stern-Gerlach experiment in which an inhomogeneous field allows for the strong measurement of the projection of the spin of spin-1/2 particles such as electrons or silver atoms has been worked out by Benitez Rodriguez et al. [22]. Here, we adapt their results to consider weak values of the spin operators in such an experiment. They considered a particle with mass $M$, which evolves in time under the Hamiltonian

$$
\hat{H}=\frac{\hat{p}_{r}^{2}}{2 M}-\mu_{c} \hat{\sigma}_{z} b(y) z
$$

such that the inhomogeneous magnetic field is assumed to be in the $z$ direction only with component $B_{z}=-b(y) z$, with $b$ denoting the inhomogeneous field strength. The spin states are eigenvalues of the $\frac{1}{2} \hat{\sigma}_{z}$ spin operator represented by its Pauli matrix and denoted as $\left|\uparrow_{z}\right\rangle$ and $\left|\downarrow_{z}\right\rangle$ with eigenvalues $\pm 1 / 2$, respectively. With this notation the eigenvalues of $\hat{\sigma}_{z}$ are \pm 1 . The parameter $\mu_{c}=g e \hbar /(2 M)$ expresses the strength of the interaction. Here, $g$ is the gyromagnetic ratio and $e$ the electron charge. In their model, the field exists everywhere and therefore the dynamics is analytically soluble. In practice, the field is limited to a spatial region of length $l$ such that if the particle is moving originally in the $y$ direction

$$
b(y)=b \theta(y) \theta(l-y),
$$

where $\theta(x)$ is the unit step function.

The coupling between the vertical $(z)$ and horizontal $(y)$ degrees of freedom makes it in principle impossible to solve the problem exactly. However, with a "reasonable" approximation, one may still present an analytic solution for the motion of the particle. Specifically, we will assume that the preselected spatial state of the particle is described by the coherent state wave packet

$\left\langle x, y, z \mid \psi_{0}\right\rangle=\frac{1}{\left(\pi d^{2}\right)^{3 / 4}} \exp \left(-\frac{x^{2}+y^{2}+z^{2}}{2 d^{2}}+i k_{y}\left(y-y_{i}\right)\right)$,

where $d$ is the spatial width of the wave packet and the subscript zero denotes the initial time. It is localized about the initial spatial point $(0,0,0)$ with mean momentum in the $y$ direction $\hbar k_{y}$. The width parameter $d$ will be chosen large enough such that the (reduced) momentum spread $\left(d k_{y}\right)^{-1} \ll$ 1. With these conditions, we may consider a classical path approximation by which the evolution of the motion in the $y$ direction may be considered to be classical. This implies that the time during which the particle feels the magnetic field is well approximated as

$$
\tau=\frac{M l}{\hbar k_{y}}
$$

The normalized preselected state is then described by the general form (with the superposition coefficients $\left|\alpha_{i}\right|^{2}+$ $\left.\left|\beta_{i}\right|^{2}=1\right)$ as

$$
\left|\Psi_{0}\right\rangle=\left|\psi_{0}\right\rangle\left[\alpha_{i}\left|\uparrow_{z}\right\rangle+\beta_{i}\left|\downarrow_{z}\right\rangle\right] .
$$

The time-dependent width is given by

$$
d_{t}^{2}=d^{2}+\frac{i \hbar t}{M}
$$

We then use reduced variables such that all lengths $(x, y, z, l)$ are reduced according to

$$
x / d \rightarrow x, \quad y / d \rightarrow y, \quad z / d \rightarrow z, \quad l / d \rightarrow l,
$$

and the time and wave numbers as

$$
\frac{\hbar t}{M d^{2}} \rightarrow t, \quad k_{y} d \rightarrow k_{y} .
$$

The physical field strength parameter $\alpha=\tau \mu_{c} b$ has dimension of $\hbar / d$ so it reduces naturally to

$$
\frac{\alpha d}{\hbar} \rightarrow \alpha
$$

With these preliminaries one finds that the time-dependent wave function at a position $y \gg l / 2$, that is, to the right of the region where the particle feels the magnetic field, is

$$
\left\langle x, y, z \mid \Psi_{t}\right\rangle=\alpha_{i} \phi_{+}(x, y, z ; t)\left|\uparrow_{z}\right\rangle+\beta_{i} \phi_{-}(x, y, z ; t)\left|\downarrow_{z}\right\rangle,
$$


with

$$
\begin{aligned}
\phi_{+}(x, y \gg l / 2, z ; t)= & {\left[\frac{1}{(1+i t) \sqrt{\pi}}\right]^{3 / 2} } \\
& \times \exp \left(-i \frac{\alpha^{2} t}{6}-\frac{x^{2}+\left(y-i k_{y}\right)^{2}+\left(z-\frac{1}{2} \alpha t\right)^{2}}{2(1+i t)}-\frac{k_{y}^{2}}{2}-i \alpha z\right)
\end{aligned}
$$

and

$$
\phi_{-}(x, y \gg l / 2, z ; t)=\phi_{+}(x, y \gg l / 2, z ; t) \exp \left(2 i \alpha z-\frac{\alpha z t}{(1+i t)}\right) .
$$

The two spatial functions are normalized, that is $\left\langle\phi_{+} \mid \phi_{+}\right\rangle=$ $\left\langle\phi_{-} \mid \phi_{-}\right\rangle=1$, but are not orthogonal:

$$
\left\langle\phi_{+} \mid \phi_{-}\right\rangle=\exp \left[-\alpha^{2}\left(1+\frac{9}{4} t^{2}\right)\right] .
$$

If the field is very strong or at long times, the overlap will effectively vanish, implying a full spatial separation of the up and down spin beams.

\section{Strong value (standard) uncertainty relation}

Before dealing with weak values, we consider the timedependent strong values for the three spins. By definition, the strong value of the $j$ th component of the Pauli matrix at time $t$ is

$$
S_{j, s}(t)=\left\langle\Psi_{t}\left|\hat{\sigma}_{j}\right| \Psi_{t}\right\rangle, \quad j=x, y, z
$$

Due to the fact that the two spin states are orthogonal to each other we have that $\left\langle\Psi_{t} \mid \Psi_{t}\right\rangle=1$ and the time-dependent strong values for the three spins are

$$
\begin{gathered}
S_{x, s}(t)=\left\langle\phi_{-} \mid \phi_{+}\right\rangle\left(\beta_{i}^{*} \alpha_{i}+\alpha_{i}^{*} \beta_{i}\right), \\
S_{y, s}(t)=i\left\langle\phi_{-} \mid \phi_{+}\right\rangle\left(\beta_{i}^{*} \alpha_{i}-\alpha_{i}^{*} \beta_{i}\right), \\
S_{z, s}(t)=\left|\alpha_{i}\right|^{2}-\left|\beta_{i}\right|^{2} .
\end{gathered}
$$

If, for example, we choose $\alpha_{i}=\frac{1}{\sqrt{2}}, \beta_{i}=\frac{1}{\sqrt{2}} \exp \left(i \chi_{i}\right)$, then the mean value of the vertical spin vanishes as expected. Since we have not postselected any state, the positive and negative spin beams have the same probability and the mean value of the vertical spin vanishes. If we choose the phase $\chi_{i}=0, \pi$, then the initial state is an eigenstate of the $\hat{\sigma}_{x}$ operator and the strong value of the spin in the $x$ direction is equal to $\left\langle\phi_{-} \mid \phi_{+}\right\rangle$.

The (cyclic) commutation and anticommutation relations of the Pauli matrices are

$$
\left[\hat{\sigma}_{x}, \hat{\sigma}_{y}\right]=2 i \hat{\sigma}_{z}, \quad\left\{\hat{\sigma}_{x}, \hat{\sigma}_{y}\right\}=0,
$$

so that the strong value (Schrödinger) uncertainty relation is

$$
\begin{aligned}
& \left\langle\Psi_{t}\left|\Delta \hat{\sigma}_{x}^{2}\right| \Psi_{t}\right\rangle\left\langle\Psi_{t}\left|\Delta \hat{\sigma}_{y}^{2}\right| \Psi_{t}\right\rangle \\
& \quad=\left[\left\langle\Psi_{t}\left|\hat{\sigma}_{x}^{2}\right| \Psi_{t}\right\rangle-\left\langle\Psi_{t}\left|\hat{\sigma}_{x}\right| \Psi_{t}\right\rangle^{2}\right]\left[\left\langle\Psi_{t}\left|\hat{\sigma}_{y}^{2}\right| \Psi_{t}\right\rangle-\left\langle\Psi_{t}\left|\hat{\sigma}_{y}\right| \Psi_{t}\right\rangle^{2}\right] \\
& \quad \geqslant-\frac{1}{4}\left\langle\Psi_{t}\left|\left[\hat{\sigma}_{x}, \hat{\sigma}_{y}\right]\right| \Psi_{t}\right\rangle^{2}=\left\langle\Psi_{t}\left|\hat{\sigma}_{z}\right| \Psi_{t}\right\rangle^{2} .
\end{aligned}
$$

Using the notation $\beta_{i}=\beta \exp (i b), \alpha_{i}=\alpha \exp (i a)$, the uncertainty relation Eq. (51) takes the form

$$
\begin{aligned}
& \left\langle\Psi_{t}\left|\Delta \hat{\sigma}_{x}^{2}\right| \Psi_{t}\right\rangle\left\langle\Psi_{t}\left|\Delta \hat{\sigma}_{y}^{2}\right| \Psi_{t}\right\rangle-\left\langle\Psi_{t}\left|\hat{\sigma}_{z}\right| \Psi_{t}\right\rangle^{2} \\
& \quad=4 \alpha^{2} \beta^{2}\left(1-\left\langle\phi_{-} \mid \phi_{+}\right\rangle^{2}+\left\langle\phi_{-} \mid \phi_{+}\right\rangle^{4} \sin ^{2}[2(a-b)]\right) \geqslant 0
\end{aligned}
$$

and one notes that the RHS is positive, since $\left\langle\phi_{-} \mid \phi_{+}\right\rangle \leqslant 1$.

\section{Weak value uncertainty relation}

To study the time-averaged weak value uncertainty relation one must choose a postselected state. We assume a screen at $y=y_{s} \gg l / 2$, that is, the postselected coordinate in the $y$ direction is chosen far to the right of the applied field. The spatial postselected state in the $x$ and $z$ direction will be denoted as $\left|\phi_{f}(x, z)\right\rangle$ so that the postselected state takes the form

$$
\left|\Phi_{f}\right\rangle=\left|\phi_{f}, y_{s}\right\rangle\left[\alpha_{f}\left|\uparrow_{z}\right\rangle+\beta_{f}\left|\downarrow_{z}\right\rangle\right] .
$$

The weak values of the three spin operators are then

$$
\begin{aligned}
& S_{x, w}=\frac{\left\langle\Phi_{f}\left|\hat{\sigma}_{x}\right| \Psi_{t}\right\rangle}{\left\langle\Phi_{f} \mid \Psi_{t}\right\rangle}=\frac{\beta_{f}^{*} \alpha_{i}+\alpha_{f}^{*} \beta_{i} R\left(y_{s}\right)}{\alpha_{f}^{*} \alpha_{i}+\beta_{f}^{*} \beta_{i} R\left(y_{s}\right)}, \\
& S_{y, w}=\frac{\left\langle\Phi_{f}\left|\hat{\sigma}_{y}\right| \Psi_{t}\right\rangle}{\left\langle\Phi_{f} \mid \Psi_{t}\right\rangle}=i \frac{\beta_{f}^{*} \alpha_{i}-\alpha_{f}^{*} \beta_{i} R\left(y_{s}\right)}{\alpha_{f}^{*} \alpha_{i}+\beta_{f}^{*} \beta_{i} R\left(y_{s}\right)}, \\
& S_{z, w}=\frac{\left\langle\Phi_{f}\left|\hat{\sigma}_{z}\right| \Psi_{t}\right\rangle}{\left\langle\Phi_{f} \mid \Psi_{t}\right\rangle}=\frac{\alpha_{f}^{*} \alpha_{i}-\beta_{f}^{*} \beta_{i} R\left(y_{s}\right)}{\alpha_{f}^{*} \alpha_{i}+\beta_{f}^{*} \beta_{i} R\left(y_{s}\right)},
\end{aligned}
$$

where $R\left(y_{s}\right)=\frac{\left\langle\phi_{f}, y_{s} \mid \phi_{-}\right\rangle}{\left\langle\phi_{f}, y_{s} \mid \phi_{+}\right\rangle}$is the ratio of the two overlaps. It may be expressed in terms of the weak spin value in the $z$ direction as

$$
R\left(y_{s}\right)=\frac{\alpha_{f}^{*} \alpha_{i}\left[1-S_{z, w}\right]}{\beta_{f}^{*} \beta_{i}\left[1+S_{z, w}\right]} .
$$

This implies a linear relation between the weak values in the $x$ and $y$ directions and the vertical direction:

$$
\begin{aligned}
& S_{x, w}=\frac{1}{2}\left[\left(\frac{\beta_{f}^{*}}{\alpha_{f}^{*}}+\frac{\alpha_{f}^{*}}{\beta_{f}^{*}}\right)+\left(\frac{\beta_{f}^{*}}{\alpha_{f}^{*}}-\frac{\alpha_{f}^{*}}{\beta_{f}^{*}}\right) S_{z, w}\right], \\
& S_{y, w}=-\frac{i}{2}\left[\left(\frac{\alpha_{f}^{*}}{\beta_{f}^{*}}-\frac{\beta_{f}^{*}}{\alpha_{f}^{*}}\right)-\left(\frac{\alpha_{f}^{*}}{\beta_{f}^{*}}+\frac{\beta_{f}^{*}}{\alpha_{f}^{*}}\right) S_{z, w}\right]
\end{aligned}
$$


and therefore also a linear relation for the fluctuations of the spins such that

$$
\Delta S_{x, w}=i \frac{\left(\alpha_{f}^{* 2}-\beta_{f}^{* 2}\right)}{\left(\alpha_{f}^{* 2}+\beta_{f}^{* 2}\right)} \Delta S_{y, w} .
$$

As in the case of the position and momentum operators, this linear relation leads to the conclusion that the weak value uncertainty principle does not pose any restriction on the weak values of the operators $\sigma_{x}$ and $\sigma_{y}$ even though they do not commute and the postselected states are not eigenfunctions of either of them.

The weak value time-averaged commutator and anticommutator are found to be

$$
\begin{gathered}
\left\langle\left[\Delta S_{x, w}^{\dagger}, \Delta S_{y, w}\right]\right\rangle=\frac{i}{2}\left\langle\left|\Delta S_{z, w}\right|^{2}\right\rangle\left[\frac{\left|\beta_{f}\right|^{2}-\left|\alpha_{f}\right|^{2}}{\left|\alpha_{f}\right|^{2}\left|\beta_{f}\right|^{2}}\right], \\
\left\langle\left\{\Delta S_{x, w}^{\dagger}, \Delta S_{y, w}\right\}\right\rangle=\frac{i}{2}\left\langle\left|\Delta S_{z, w}\right|^{2}\right\rangle\left[\frac{\beta_{f}^{2} \alpha_{f}^{* 2}-\alpha_{f}^{2} \beta_{f}^{* 2}}{\left|\alpha_{f}\right|^{2}\left|\beta_{f}\right|^{2}}\right] .
\end{gathered}
$$

These are quite different from their strong value analogs as derived from Eqs. (50). The weak value commutator may vanish, if the postselected state is chosen (as is often the case) such that $\left|\beta_{f}\right|^{2}=\left|\alpha_{f}\right|^{2}$. With these results it is also straightforward to find that

$$
\left\langle\left|\Delta S_{x, w}\right|^{2}\right\rangle\left\langle\left|\Delta S_{y, w}\right|^{2}\right\rangle=\frac{\left|\left(\beta_{f}^{4}-\alpha_{f}^{4}\right)\right|^{2}}{16\left|\alpha_{f} \beta_{f}\right|^{4}}\left\langle\left|\Delta S_{z, w}\right|^{2}\right\rangle^{2},
$$

showing that if the standard deviation from the time-averaged weak value of the vertical spin vanishes, then there is no constraint on the analogous standard deviation of the weak values in the $x$ and $y$ directions.

\section{Time-spin uncertainty relation}

It is also illuminating to study the relationship between the time-averaged variance of the weak value of any of the spins and the variance of the time it takes the particle passing through the Stern-Gerlach machine to reach the final screen. Here, the weak value of the time is defined as in Eq. (19) and the weak values of the spin as in Eqs. (54)-(56). To apply the weak value uncertainty principle as in Eq. (17) we first note that the time probability distribution at the postselected state as chosen in Eq. (53) is

$$
P_{\Psi}(t ; \Phi)=\frac{\left|\alpha_{f}^{*} \alpha_{i} \int_{-\infty}^{\infty} d x d z \phi_{f}^{*}\left(x, z, y_{s}\right) \phi_{+}\left(x, y_{s}, z ; t\right)+\beta_{f}^{*} \beta_{i} \int_{-\infty}^{\infty} d x d z \phi_{f}^{*}\left(x, z, y_{s}\right) \phi_{-}\left(x, y_{s}, z ; t\right)\right|^{2}}{N(\Phi ; \Psi)} .
$$

The time-spin uncertainty relation is then readily seen to be

$$
\left\langle\left|\Delta S_{j, w}\right|^{2}\right\rangle\left\langle|\Delta t|^{2}\right\rangle \geqslant\left|\left\langle t S_{j, w}\right\rangle\right|^{2},
$$

with $j=x, y, z$. This is a nontrivial statement since it shows that the variance of the weak spin is directly proportional to its time modulated magnitude. In other words, large anomalous values of the spin lead to large variance of the weak spin values and thus imply larger experimental averaging needed than for "normal" spin values.

\section{SPECIFIC APPLICATIONS}

\section{A. Potential scattering}

\section{Coordinate-momentum uncertainty}

To exemplify the consequences of the weak value uncertainty principle, we consider in some detail the scattering of a particle through a potential barrier, where, for the sake of simplicity, the potential $V(q)$ vanishes as $q \rightarrow \pm \infty$. For an incident momentum $p_{i}$ of a particle with mass $M$ from the left, the transmission amplitude through the barrier is denoted by $T\left(p_{i}\right)$. We choose the preselected state $\langle x \mid \Psi\rangle$ to be a coherent state as in Eq. (26) such that the point $x_{i}$ is sufficiently far to the left of the potential so that the potential is negligible. This implies that $\Gamma x_{i}^{2} \gg 1$. As shown in [15] the time evolved wave function in the region far to the right of the potential may be approximated by steepest descent to be

$$
\begin{aligned}
\langle x \mid \Psi(t)\rangle \simeq & \left(\frac{\Gamma M^{2}}{\pi(M+i t \hbar \Gamma)^{2}}\right)^{\frac{1}{4}} T\left(\frac{M p_{i}-i \hbar \Gamma M\left(x_{i}-x\right)}{(M+i t \hbar \Gamma)}\right) \\
& \times \exp \left(-\frac{p_{i}^{2}}{2 \hbar^{2} \Gamma}+\frac{M \Gamma}{2} \frac{\left[i\left(x_{i}-x\right)-\frac{p_{i}}{\hbar \Gamma}\right]^{2}}{[M+i t \hbar \Gamma]}\right) .
\end{aligned}
$$

We then choose the postselected state to be a Gaussian localized about the point $x_{f}$ sufficiently far out to the right of the potential so that it does not overlap with the potential, with vanishing mean momentum, and to simplify the width parameter $\Gamma_{f} \gg \Gamma$ is chosen sufficiently large such that the postselected state effectively localizes the particle at the point $x_{f}$. Within the steepest descent approximation, using the notation

$$
\Delta x=x_{f}-x_{i},
$$

the weak value of the momentum is

$$
\begin{aligned}
p_{w}\left(\Phi_{f} ; \Psi_{t}\right) \simeq & \frac{M^{2} p_{i}+\hbar^{2} \Gamma^{2} M \Delta x t}{\left[M^{2}+t^{2} \hbar^{2} \Gamma^{2}\right]} \\
& +i \hbar M \Gamma \frac{p_{i} t-M \Delta x}{\left[M^{2}+t^{2} \hbar^{2} \Gamma^{2}\right]}+O\left(\frac{\Gamma}{\Gamma_{f}}\right)
\end{aligned}
$$

and the weak value of the coordinate [see Eq. (27)] is

$$
x_{w}\left(\Phi ; \Psi_{t}\right)=x_{f}+O\left(\frac{\Gamma}{\Gamma_{f}}\right) .
$$


Since the weak value of the coordinate is time independent, it has no uncertainty associated with it; therefore, the variance of the weak value of the momentum can, by suitable choice of the width parameter of the preselected state, be made arbitrarily small.

The steepest-descent approximation for the time probability distribution is readily found to be (ignoring terms of the order of $\frac{\Gamma}{\Gamma_{f}}$ )

$$
\begin{aligned}
\frac{\left|\left\langle\Phi_{f} \mid \Psi(t)\right\rangle\right|^{2}}{\int_{0}^{\infty} d t\left|\left\langle\Phi_{f} \mid \Psi(t)\right\rangle\right|^{2}} \simeq & p_{i}\left(\frac{p_{i}^{2} \Gamma}{\pi\left[M^{2} p_{i}^{2}+M^{2} \Delta x^{2} \hbar^{2} \Gamma^{2}\right]}\right)^{\frac{1}{2}} \\
& \times \exp \left(-\frac{p_{i}^{2} \Gamma\left(M \Delta x-p_{i} t\right)^{2}}{\left[M^{2} p_{i}^{2}+M^{2} \Delta x^{2} \hbar^{2} \Gamma^{2}\right]}\right),
\end{aligned}
$$

so that the time-averaged mean of the momentum is within the steepest-descent approximation:

$$
\left\langle p_{w}\left(\Phi_{f} ; \Psi_{t}\right)\right\rangle \simeq p_{i}+O\left(\frac{\Gamma}{\Gamma_{f}}\right),
$$

while the time-averaged mean of the weak value of the coordinate is of course

$$
\left\langle x_{w}\left(\Phi ; \Psi_{t}\right)\right\rangle \simeq x_{f}+O\left(\frac{\Gamma}{\Gamma_{f}}\right) .
$$

The variance of the weak value of the momentum is readily seen to be

$$
\left\langle\left|p_{w}\left(\Phi_{f} ; \Psi_{t}\right)\right|^{2}\right\rangle-\left|\left\langle p_{w}\left(\Phi_{f} ; \Psi_{t}\right)\right\rangle\right|^{2} \simeq \frac{\hbar^{2} \Gamma}{2}
$$

and as noted before can be made arbitrarily small by choosing the width parameter of the initial state to be sufficiently small.

\section{Kinetic-energy uncertainty}

Within the same framework as above, the weak value of the kinetic energy is readily seen to be

$$
T_{w}\left(\Phi_{f} ; \Psi_{t}\right) \simeq \frac{1}{2}\left[\frac{\hbar^{2} \Gamma}{[M+i t \hbar \Gamma]}-\frac{M\left(i p_{i}-\hbar \Gamma \Delta x\right)^{2}}{[M+i t \hbar \Gamma]^{2}}\right]
$$

and its time-averaged mean is thus complex:

$$
\begin{aligned}
\left\langle T_{w}\left(\Phi_{f} ; \Psi_{t}\right)\right\rangle \simeq & \frac{p_{i}^{2}}{2 M}\left[1+\frac{\hbar^{2} \Gamma}{\left[p_{i}^{2}+\Delta x^{2} \hbar^{2} \Gamma^{2}\right]}\right] \\
& -\frac{i \hbar p_{i} \hbar^{2} \Gamma^{2} \Delta x}{M\left[p_{i}^{2}+\Delta x^{2} \hbar^{2} \Gamma^{2}\right]} .
\end{aligned}
$$

In the limit that the distance between the initial and final points becomes very large, such that $\Gamma \Delta x^{2} \gg p_{i}^{2} /\left(\hbar^{2} \Gamma\right)$ and $\Gamma \Delta x^{2} \gg 1$, one finds that

$$
\begin{aligned}
\left\langle T_{w}\left(\Phi_{f} ; \Psi_{t}\right)\right\rangle \simeq & \frac{p_{i}^{2}}{2 M}\left[1+\frac{1}{\Delta x^{2} \Gamma}\right]-\frac{i \sqrt{\hbar^{2} \Gamma} p_{i}}{M \sqrt{\Gamma \Delta x^{2}}} \\
& +O\left(\frac{p_{i}^{2}}{\sqrt{\Gamma \Delta x^{2}} \Delta x^{2} \hbar^{2} \Gamma^{2}}\right) .
\end{aligned}
$$

With some further algebra one also finds that within the steepest-descent estimate the variance of the weak value of the kinetic energy is

$$
\begin{aligned}
& \left\langle\left|T_{w}\left(\Phi_{f} ; \Psi_{t}\right)\right|^{2}\right\rangle-\left|\left\langle T_{w}\left(\Phi_{f} ; \Psi_{t}\right)\right\rangle\right|^{2} \\
& \simeq \frac{2 \hbar^{2} \Gamma p_{i}^{6}\left(\Delta x^{2} \hbar^{2} \Gamma^{2}\right)}{M^{2}\left[p_{i}^{2}+\Delta x^{2} \hbar^{2} \Gamma^{2}\right]^{3}}+\frac{\hbar^{2} \Gamma\left(\hbar^{2} \Gamma+2 p_{i}^{2}\right)^{2}}{8 M^{2}\left[p_{i}^{2}+\Delta x^{2} \hbar^{2} \Gamma^{2}\right]} \\
& \quad+\frac{\hbar^{6} \Gamma^{3} \Gamma \Delta x^{2} p_{i}^{2}}{2 M^{2}\left[p_{i}^{2}+\Delta x^{2} \hbar^{2} \Gamma^{2}\right]^{2}}
\end{aligned}
$$

As before in the limit that $\Gamma \Delta x^{2} \gg p_{i}^{2} /\left(\hbar^{2} \Gamma\right)$ and $\Gamma \Delta x^{2} \gg 1$ this simplifies to

$$
\begin{aligned}
& \left\langle\left|T_{w}\left(\Phi_{f} ; \Psi_{t}\right)\right|^{2}\right\rangle-\left|\left\langle T_{w}\left(\Phi_{f} ; \Psi_{t}\right)\right\rangle\right|^{2} \\
& \simeq \frac{1}{\Gamma \Delta x^{2}}\left(\frac{\hbar^{4} \Gamma^{2}}{8 M^{2}}+\frac{p_{i}^{4}}{2 M^{2}}+\frac{\hbar^{2} \Gamma p_{i}^{2}}{M^{2}}\right),
\end{aligned}
$$

demonstrating that one should expect that the variance in the kinetic energy vanishes when the distance between the initial and final points is sufficiently large.

It remains to consider the mean time and its variance. Within the steepest-descent approximation one has that the mean time and its variance are given by

$$
\begin{gathered}
\langle t\rangle=\frac{M \Delta x}{p_{i}} \\
\left\langle(t-\langle t\rangle)^{2}\right\rangle=\frac{\left[M^{2} p_{i}^{2}+M^{2} \Delta x^{2} \hbar^{2} \Gamma^{2}\right]}{2 \Gamma p_{i}^{4}}
\end{gathered}
$$

and one notes that when $\Gamma \Delta x^{2} \gg p_{i}^{2} /\left(\hbar^{2} \Gamma\right)$ and $\Gamma \Delta x^{2} \gg 1$, the time variance diverges. The product of the kinetic energy and time variances is

$$
\begin{gathered}
{\left[\left\langle\left|T_{w}\left(\Phi_{f} ; \Psi_{t}\right)\right|^{2}\right\rangle-\left|\left\langle T_{w}\left(\Phi_{f} ; \Psi_{t}\right)\right\rangle\right|^{2}\right]\left\langle(t-\langle t\rangle)^{2}\right\rangle} \\
\simeq \frac{\hbar^{2} p_{i}^{2}\left(\Delta x^{2} \hbar^{2} \Gamma^{2}\right)}{\left[p_{i}^{2}+\Delta x^{2} \hbar^{2} \Gamma^{2}\right]^{2}}+\frac{\hbar^{2}\left(\hbar^{2} \Gamma+2 p_{i}^{2}\right)^{2}}{16 p_{i}^{4}} \\
\quad+\frac{\hbar^{6} \Gamma^{3} \Delta x^{2}}{4 p_{i}^{2}\left[p_{i}^{2}+\Delta x^{2} \hbar^{2} \Gamma^{2}\right]}
\end{gathered}
$$

and in the limit that $\Gamma \Delta x^{2} \gg p_{i}^{2} /\left(\hbar^{2} \Gamma\right)$ and $\Gamma \Delta x^{2} \gg 1$ we find that

$$
\begin{gathered}
{\left[\left\langle\left|T_{w}\left(\Phi_{f} ; \Psi_{t}\right)\right|^{2}\right\rangle-\left|\left\langle T_{w}\left(\Phi_{f} ; \Psi_{t}\right)\right\rangle\right|^{2}\right]\left\langle(t-\langle t\rangle)^{2}\right\rangle} \\
\simeq \frac{\hbar^{2}}{4}\left(1+\frac{2 \hbar^{2} \Gamma}{p_{i}^{2}}+\frac{\hbar^{4} \Gamma^{2}}{4 p_{i}^{4}}\right) \geqslant \frac{\hbar^{2}}{4}
\end{gathered}
$$

in accordance with the weak value time-energy uncertainty relation.

In summary, from the analysis of time-averaged weak values one concludes that, in a scattering experiment, one may know the precise value of the momentum and the kinetic energy of a particle as it hits the screen, that is, at a known location. However, as the precision increases, the variance in the time of arrival of the particle at the screen increases, and the product of the kinetic energy and time variances is limited by the standard uncertainty relation. 


\section{B. Stern-Gerlach experiment}

To demonstrate the results more clearly for the SternGerlach setup we present some numerical results. For this purpose we have to specify the postselected state. The field splits the beam into two parts: one localized on the positive $z$ axis the other on the negative. We will choose the spatial part of the postselected state to be localized on the positive $z$ axis at $z=z_{0}$, where $z_{0}$ is at the center of the positive beam found on a screen located at and about $x=0$. We then choose the final spatial state to be a Gaussian centered about $z_{0}$ :

$$
\left\langle x, z \mid \phi_{f}\right\rangle=\left(\frac{1}{\pi}\right)^{1 / 4} \exp \left(-\frac{x^{2}+\left(z-z_{0}\right)^{2}}{2}\right) .
$$

The overlap integrals involve a Gaussian integration over the $x$ and $z$ coordinates:

$$
\left\langle\phi_{f}, y_{s} \mid \phi_{+}\right\rangle=\frac{2}{\sqrt{1+i t}(2+i t)} \exp \left(-i \frac{\alpha^{2} t}{6}-\frac{\left(y-i k_{y}\right)^{2}}{2(1+i t)}-\frac{\left(z_{0}-\frac{1}{2} \alpha t-i \alpha\right)^{2}}{2(2+i t)}-\frac{k_{y}^{2}+\alpha^{2}}{2}-i \alpha z_{0}\right)
$$

and the ratio of the two overlaps [see text preceding Eq. (57)] is

$$
R\left(y_{s}\right)=\exp \left(i \eta_{t}-Y_{t}\right), \quad \eta_{t}=\frac{\alpha z_{0}\left(1+\frac{3}{4} t^{2}\right)}{\left(1+\frac{t^{2}}{4}\right)}, \quad Y_{t}=\frac{\alpha z_{0} t}{\left(1+\frac{t^{2}}{4}\right)}
$$

The time-dependent densities are then

$$
\begin{gathered}
\left|\left\langle\phi_{f}, y_{s} \mid \phi+\right\rangle\right|^{2}=\frac{1}{\sqrt{1+t^{2}}\left(1+\frac{t^{2}}{4}\right)} \exp \left(-\frac{\left(y_{s}-k_{y} t\right)^{2}}{\left(1+t^{2}\right)}-\frac{\left(z_{0}-\alpha t\right)^{2}}{2\left(1+\frac{t^{2}}{4}\right)}-\frac{\alpha^{2}}{2}\right) \\
\equiv \frac{1}{\sqrt{1+t^{2}}\left(1+\frac{t^{2}}{4}\right)} \exp \left(-\rho(z, t)-\frac{\alpha^{2}}{2}\right), \\
\left|\left\langle\phi_{f}, y_{s} \mid \phi-\right\rangle\right|^{2}=\left|\left\langle\phi_{f}, y_{s} \mid \phi+\right\rangle\right|^{2} \exp \left(-\frac{2 \alpha t z_{0}}{\left(1+\frac{t^{2}}{4}\right)}\right) .
\end{gathered}
$$

In Fig. 1 we plot the sum of the densities as a function of the location on the vertical axis $z_{0}$ and time for a weak field [panel (a), $\alpha=1 / 2$ ] and a strong field [panel (b), $\alpha=4$ ] for $y_{s}=-y_{i}=k_{y}=10$. As discussed above, the location of the postselected state will be such that the density maximizes about the center of the Gaussian in space and time such that

$$
z_{0}=\alpha \bar{t}=\frac{\alpha y_{s}}{k_{y}} .
$$

Noting that

$$
\left.\frac{1}{2} \frac{d^{2} \rho\left(z_{0}, t\right)}{d t^{2}}\right|_{t=\bar{t}}=\left[\frac{k_{y}^{2}+y_{s}^{2}}{\left(1+\bar{t}^{2}\right)^{2}}+\frac{\alpha^{2}}{2\left(1+\frac{\bar{t}^{2}}{4}\right)}\right]
$$

one finds that within the steepest-descent estimate the normalization integral is

$$
\begin{aligned}
N(\Phi ; \Psi) & =\frac{1}{2} \int_{0}^{\infty} d t\left|\left\langle\phi_{f}, y_{s} \mid \phi_{+}\right\rangle\right|^{2}\left[1+\exp \left(-2 Y_{t}\right)\right] \\
& =\frac{1}{2} \frac{\sqrt{2 \pi\left(1+\bar{t}^{2}\right)} \exp \left(-\frac{\alpha^{2}}{2}\right)}{\sqrt{\left(1+\frac{\bar{t}^{2}}{4}\right)} \sqrt{2\left(k_{y}^{2}+y_{s}^{2}\right)\left(1+\frac{\bar{t}^{2}}{4}\right)+\alpha^{2}\left(1+\bar{t}^{2}\right)^{2}}}\left[1+\exp \left(\frac{-4 \alpha^{2} \bar{t}^{2}\left(k_{y}^{2}+y_{s}^{2}\right)}{\left[2\left(k_{y}^{2}+y_{s}^{2}\right)\left(1+\frac{\bar{t}^{2}}{4}\right)+\alpha^{2}\left(1+\bar{t}^{2}\right)^{2}\right]}\right)\right] .
\end{aligned}
$$

At this point, we will further specify the pre- and postselected states. Following the experiments of Sponar et al. [23], we choose them to be

$$
\alpha_{i}=\alpha_{f}=\frac{1}{\sqrt{2}}, \quad \beta_{i}=\frac{1}{\sqrt{2}} \exp \left(i \chi_{i}\right), \quad \beta_{f}=\frac{1}{\sqrt{2}} \exp \left(i \chi_{f}\right),
$$

where the phases $\chi_{i}$ and $\chi_{f}$ are in principle experimentally controllable phases. The weak values of the spin simplify to

$$
\begin{gathered}
S_{x, w}=\frac{\cosh \left(Y_{t}\right) \cos \left(\chi_{f}\right)+\cos \left(\chi_{i}+\eta_{t}\right)-i \sinh \left(Y_{t}\right) \sin \left(\chi_{f}\right)}{\cosh \left(Y_{t}\right)+\cos \left(\chi_{i}-\chi_{f}+\eta_{t}\right)}, \\
S_{y, w}=\frac{\cosh \left(Y_{t}\right) \sin \left(\chi_{f}\right)+\sin \left(\chi_{i}+\eta_{t}\right)+i \sinh \left(Y_{t}\right) \cos \left(\chi_{f}\right)}{\cosh \left(Y_{t}\right)+\cos \left(\chi_{i}-\chi_{f}+\eta_{t}\right)}, \\
S_{z, w}=\frac{\sinh \left(Y_{t}\right)-i \sin \left(\chi_{i}-\chi_{f}+\eta_{t}\right)}{\cosh \left(Y_{t}\right)+\cos \left(\chi_{i}-\chi_{f}+\eta_{t}\right)} .
\end{gathered}
$$


The steepest-descent estimate for the transition path time distribution is

$$
\begin{aligned}
& P_{\Psi}(t ; \Phi)=\frac{\frac{1}{2}\left|\left\langle\phi_{f}, y_{s} \mid \phi_{+}\right\rangle\right|^{2}\left[1+\exp \left(-2 Y_{t}\right)\right]}{\frac{1}{2} \int_{0}^{\infty} d t\left|\left\langle\phi_{f}, y_{s} \mid \phi_{+}\right\rangle\right|^{2}\left[1+\exp \left(-2 Y_{t}\right)\right]} \\
& \simeq \frac{1}{\sqrt{\pi}} \sqrt{\left[\frac{k_{y} y_{s}}{\bar{t}\left(1+\bar{t}^{2}\right)}+\frac{\alpha^{2}}{2\left(1+\frac{\bar{t}^{2}}{4}\right)}\right] \exp \left(-\left[\frac{k_{y} y_{s}}{\bar{t}\left(1+\bar{t}^{2}\right)}+\frac{\alpha^{2}}{2\left(1+\frac{\bar{t}^{2}}{4}\right)}\right](t-\bar{t})^{2}\right) .}
\end{aligned}
$$

We then have all the information needed to determine the time-averaged weak values and variances and thus to study the weak value uncertainties.

For implementation we choose $k_{y}=10, y_{s}=10$, so that $\bar{t}=1$. One should then distinguish between a weak field $(\alpha<1)$ and a strong field $(\alpha>1)$. In the strong-field case $Y_{\bar{t}} \gg 1$ so that in this limit Eqs. (92)-(94) imply that for all three spin values $\left|S_{j, w}\right|^{2} \rightarrow 1$ and nothing interesting occurs. The situation differs drastically when the field is weak. In panels (a)-(c) of Fig. 2 we plot the absolute value of the timeaveraged weak values of the spin in the $x, y$, and $z$ directions for $\alpha=1 / 2$ as a function of the pre- and postselected phases $\chi_{i}$ and $\chi_{f}$. When the field is weak one obtains substantial deviations from unity and the spin values can become much larger than unity.

The difference between the weak- and strong-field cases lies in the weak values, not in the time averaging. The transition path time distribution is insensitive to the field strength; it mainly reflects the distribution of momenta in the preselected state, as may be seen from Fig. 3. It is very narrow and localized about the time one would expect from the motion of a free particle whose momentum is 10 and the distance traveled is 10 . The differences between the weak and strong fields for the weak value spins reflects the central difference between the effect of strong and weak fields on the spin weak values. In a weak field, the overlap of pre- and postselected states is a sensitive function of the phases; it is not so for the strong field. This of course means that only when using (a)

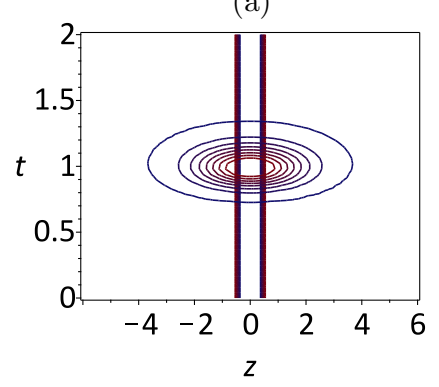

(b)

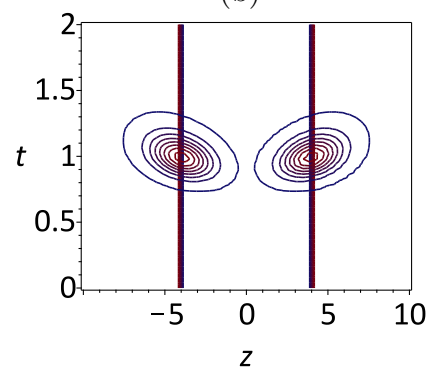

FIG. 1. Sum of the time-dependent densities in the vertical direction as obtained from addition of Eqs. (86) and (87). Panels (a) and (b) show the densities obtained for the weak and strong fields $(\alpha=1 / 2,4)$, respectively. The densities are normalized to unit height. Contours are plotted for the values $0.7,0.6,0.5,0.4,0.3,0.2,0.1,0.01$. Note the overlap of the densities in the weak field case and their separation when the field is stronger. The vertical bars denote the location of the maxima of the separate up and down densities. a weak field will one be able to discern the phases from the weak values.

The time-averaged values of the spins may be estimated using the steepest-descent approximation. The leading-order contribution is

$$
\begin{aligned}
& \left\langle S_{j, w}(t)\right\rangle \simeq S_{j, w}(\bar{t}) \\
& \quad+\left.\frac{1}{4} \frac{d^{2} S_{j, w}(t)}{d t^{2}}\right|_{t=\bar{t}}\left[\frac{k_{y} y_{s}}{\bar{t}\left(1+\bar{t}^{2}\right)}+\frac{\alpha^{2}}{2\left(1+\frac{\bar{t}^{2}}{4}\right)}\right]^{-1}
\end{aligned}
$$

The variance of the time-averaged weak spin values may be approximated as

$$
\begin{gathered}
\left\langle S_{j, w}(t) S_{j, w}^{*}(t)\right\rangle-\left|\left\langle S_{j, w}(t)\right\rangle\right|^{2} \simeq\left|\frac{d S_{j, w}(t)}{d t}\right|_{t=\bar{t}}^{2}\left\langle(t-\bar{t})^{2}\right\rangle \\
=\frac{1}{2}\left|\frac{d S_{j, w}(t)}{d t}\right|_{t=\bar{t}}^{2}\left[\frac{k_{y} y_{s}}{\bar{t}\left(1+\bar{t}^{2}\right)}+\frac{\alpha^{2}}{2\left(1+\frac{\bar{t}^{2}}{4}\right)}\right]^{-1} \cdot
\end{gathered}
$$

To make further progress we check the accuracy of the steepest-descent approximation to the time-averaged weak values of the absolute value of the three spins. In Fig. 3 we show that the steepest-descent approximation to the transition path time distribution is quite accurate. Furthermore, we found that the relative error for the time-averaged spin weak values obtained using the leading-order term only for the steepest-descent time average of the spins, $S_{j, w}(\bar{t})$, as compared to the numerically exact results is in all cases $\sim 0.5 \%$ or lower. This enables us to obtain the standard deviation for the time-averaged weak values, defined as $\sigma_{j}=$ $\sqrt{\left\langle\Delta S_{j, w}^{2}\right\rangle}$ from the steepest-descent estimate as in Eq. (59). The results for the weak-field $(\alpha=1 / 2)$ case are shown in Fig. 4. As anticipated from the general weak value uncertainty principle, we find that when the weak values are much larger than unity in magnitude there also is a relatively large standard deviation of $\sim 30 \%$, confirming that for anomalous weak values one needs more averaging of the experimental signal to determine them. This is further clarified when considering the ratio of the standard deviation to the magnitude of the spin as shown in the weak field $(\alpha=1 / 2)$ case in Fig. 5. 
(a)

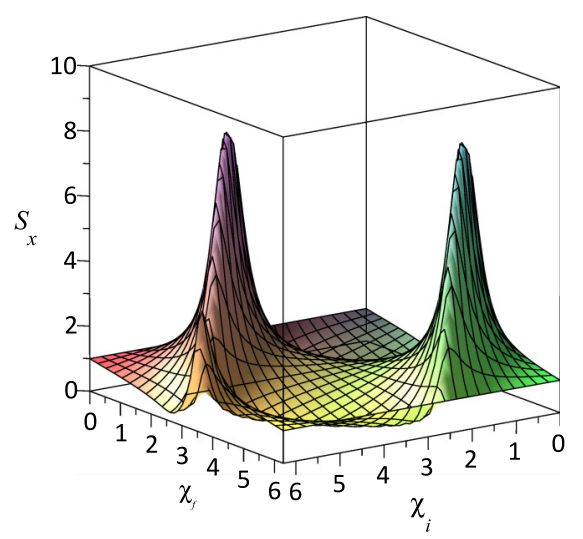

(b)

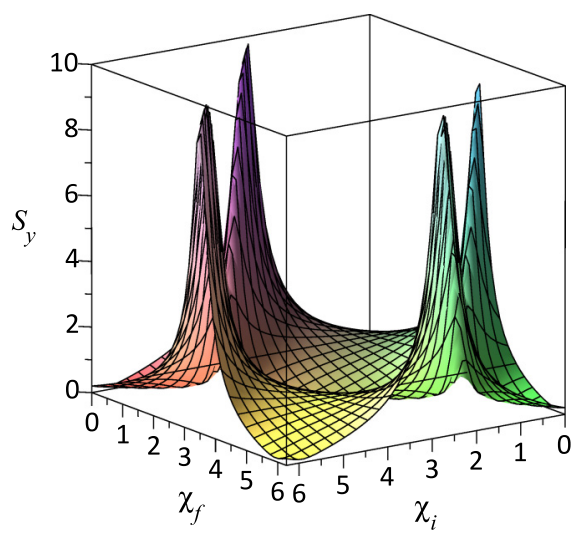

(c)

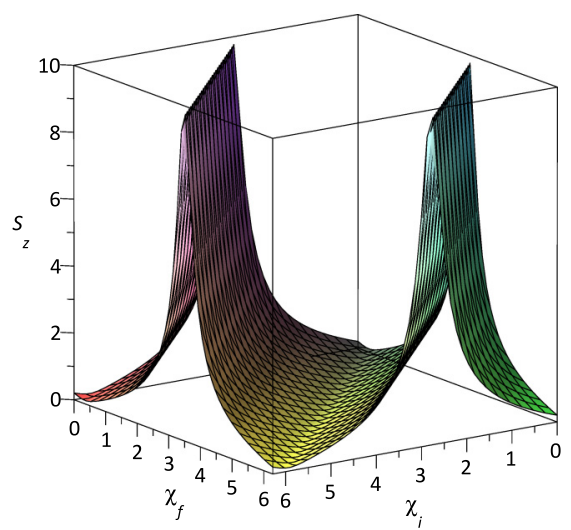

FIG. 2. Pre- and postselected phase dependence of the time-averaged absolute values of the spin weak values for a weak field $(\alpha=1 / 2)$. Panels (a)-(c) show the results for the $x, y$, and $z$ components, respectively. Note that when the field is weak the absolute magnitude of the time-averaged weak values can become much larger than unity.

Some further comments about the time-averaged weak values are in order. Denoting

$$
w=\chi_{i}-\chi_{f},
$$

one finds from Eq. (94) that within the steepest-descent (SD) approximation

$$
\left|\left\langle S_{z, w}\right\rangle_{\mathrm{SD}}\right|^{2}=\frac{\cosh \left(Y_{\bar{i}}\right)-\cos \left(w+\eta_{\bar{t}}\right)}{\cosh \left(Y_{\bar{t}}\right)+\cos \left(w+\eta_{\bar{i}}\right)} .
$$

The value of $w$ that maximizes the weak value in the vertical direction is

$$
w+\eta_{t}=(2 m+1) \pi,
$$

so that

$$
\max \left|\left\langle S_{z, w}\right\rangle_{\mathrm{SD}}\right|^{2}=\frac{\cosh \left(Y_{\bar{i}}\right)+1}{\cosh \left(Y_{\bar{i}}\right)-1}=\operatorname{coth}^{2}\left(\frac{Y_{\bar{i}}}{2}\right) .
$$

(a)

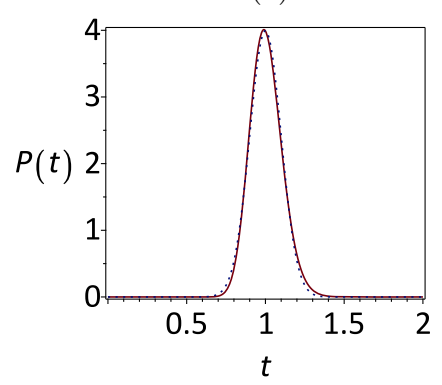

(b)

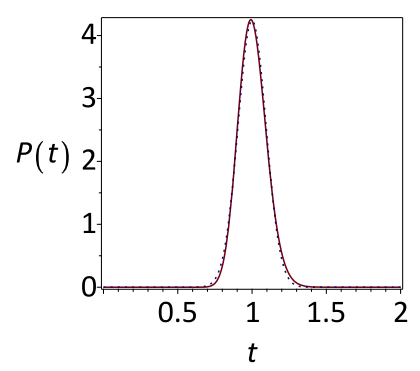

FIG. 3. Transition path time distribution for a weak magnetic field $[\alpha=1 / 2$, panel (a) $]$ and a strong one $[\alpha=4$, panel (b)]. The solid and dotted lines in each panel are respectively the numerically exact transition path time distribution and its steepestdescent estimate [Eq. (58)]. Note the accuracy of the steepest-descent approximation.
As the field strength $\alpha$ grows weaker, $Y_{\bar{i}}$ becomes smaller and the weak value grows; that is why when $\alpha=1 / 2$ we find the maximal value of $\simeq 10$ for the magnitude of the weak value.

\section{DISCUSSION AND CONCLUSION}

A weak value uncertainty relation was derived for two not necessarily Hermitian operators which do not commute. For this purpose, weak value operators were defined and their basic algebra presented. The uncertainty relation was obtained by noting that, in principle, weak values may be time dependent. The time-averaged means and variances of the weak values were obtained through a suitably defined transition path time distribution. The weak value uncertainty relation relates the variances of the weak value operators to the mean values of their commutators and anticommutators. The resulting relation depends not only on the operators and their commutation relations but also on the specific choice of preand postselected states. The resulting weak value uncertainty relation can be considered as a direct analog of the strong value (Schrödinger) uncertainty relation. An important consequence of the weak value uncertainty relation is that it is possible to know, with high accuracy, the simultaneous mean weak values of noncommuting operators even when the postselected state is not an eigenfunction of either of them by employing a judicious choice of the pre- and postselected states.

To illustrate the utility of the uncertainty relation several examples have been analyzed. First, the weak value timeenergy uncertainty relation has been evaluated and reduced to the standard value of $\hbar^{2} / 4$ when initially the overlap of the pre- and postselected states vanishes. Second, due to the proportionality found between the weak values of the momentum and coordinate when the postselected state is a coherent state, both quantities can be known simultaneously with arbitrary precision even though the two operators themselves do not commute and the coherent states are not their eigenfunctions. Third, the weak value coordinate-kinetic-energy uncertainty 
(a)

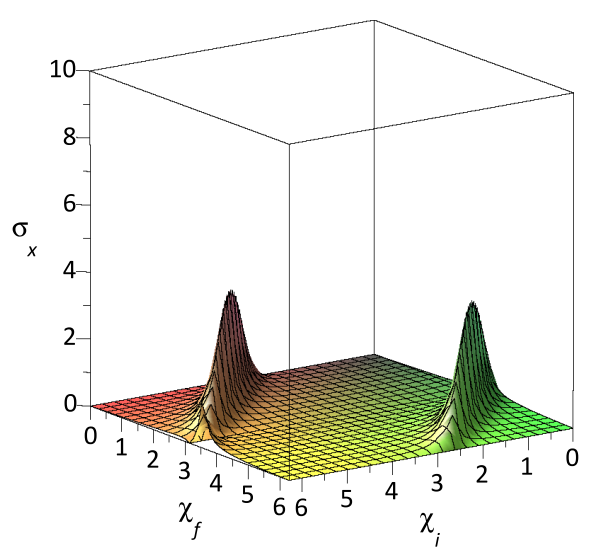

(b)

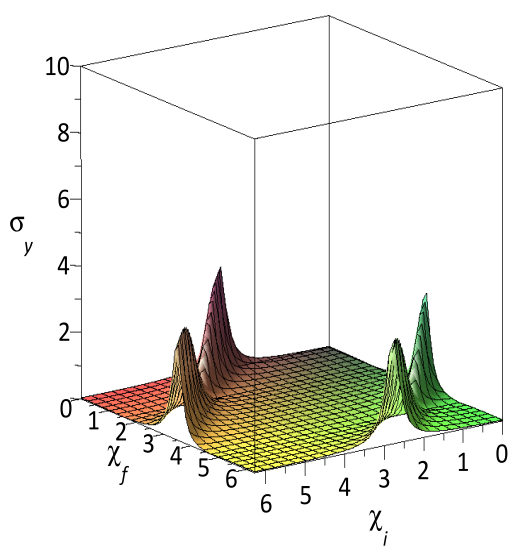

(c)

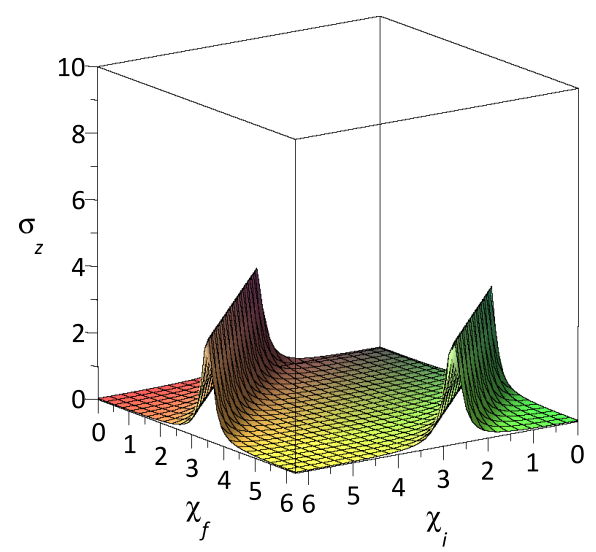

FIG. 4. Standard deviation of the time-averaged spin weak values for a weak field. Panels (a)-(c) show the results for $\sigma_{x}, \sigma_{y}$, and $\sigma_{z}$, respectively, for a field strength of $\alpha=1 / 2$.

relation when the postselected state is a coherent state shows that uncertainty in the coordinate also leads to an uncertainty in the kinetic energy. And, finally, the same analysis has been carried out for cyclic operators such as the spin operators when analyzing the Stern-Gerlach experiment. The fluctuations of the weak values of the spin also display a proportionality implying that the uncertainty principle does not impose any restriction on the spin weak values even if their corresponding operators do not commute. The weak value uncertainty relation leads to the observation that the variance of the spin weak value is proportional to its magnitude. This means that for anomalously large weak values, the variance is also large, so that from an experimental point of view more measurements on the system have to be carried out in order to obtain the precise mean anomalously large weak value as compared to the standard spin values.

The time averaging of weak values which lies at the heart of the weak value uncertainty relation has not been considered previously. As laid out in the original paper by Aharonov and co-workers [4], the weak measurement of an operator is implemented by coupling the system to the measuring device through the same operator. As a result, the time evolution operator commutes with the operator whose weak value is measured so that the weak value becomes time independent. Under such conditions, the time averaging considered in the present paper becomes trivial and unnecessary. However, our analysis of the Stern-Gerlach experiment, based on the scenario presented in Ref. [4], shows that in actuality one cannot isolate the spin component from the spatial component so that the time evolution becomes nontrivial and impacts the measured weak values. In this sense, one should consider the original scenario suggested in Ref. [4] as expressing the time-averaged spin weak values. As shown, these too can be anomalously large, verifying the results of Aharonov et al. (a)

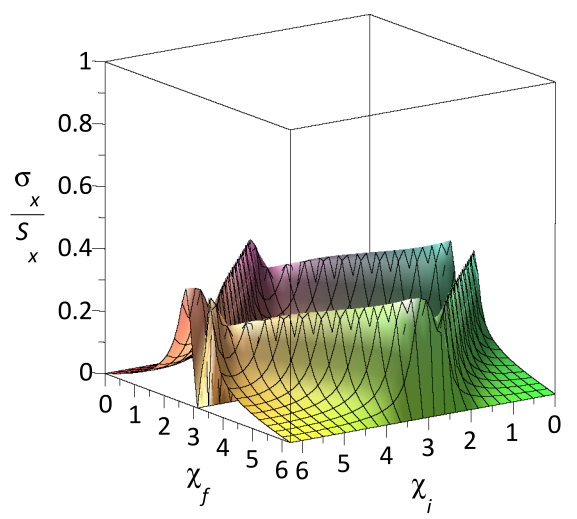

(b)

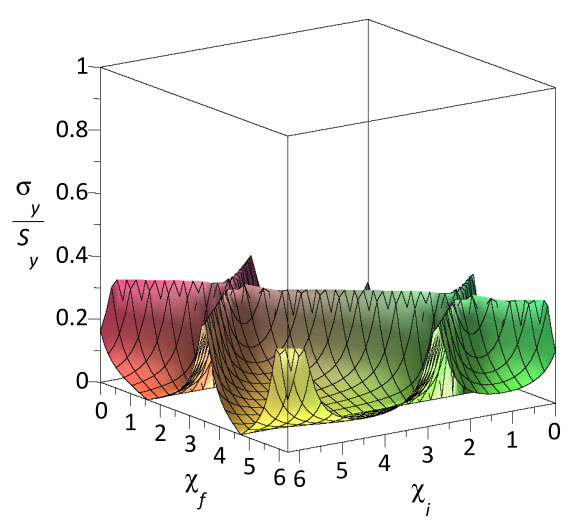

(c)

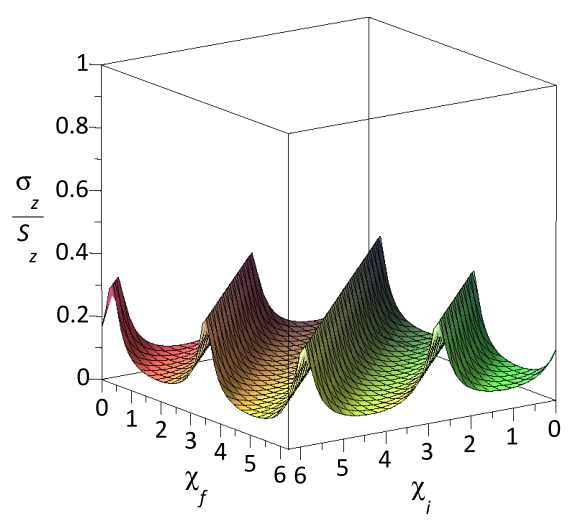

FIG. 5. Ratio of the standard deviation of the time-averaged spin weak values for a strong field to the magnitude of the spin weak value. Panels (a)-(c) show the results for the $x, y$, and $z$ directions, respectively. 


\section{ACKNOWLEDGMENTS}

We thank E. Cohen and L. Vaidman for illuminating discussions. This work was supported by a grant from the Israel Science Foundation and was partially supported by Grant No. FIS2017-83473-C2-1-P from the Ministerio de Ciencia, Innovación y Universidades (Spain).

\section{APPENDIX: DERIVATION OF THE TIME-AVERAGED WEAK VALUE UNCERTAINTY RELATION}

The purpose of this Appendix is to show in more detail how Eq. (17) is derived from the uncertainty relation given in Eq. (16). The commutator and anticommutator of the two postselected operators $\hat{A}_{\Phi}$ and $\hat{B}_{\Phi}$ [see the definition of postselected operators given in Eq. (2)] are respectively

$$
\begin{aligned}
& {\left[\Delta \hat{B}_{\Phi}^{\dagger}, \Delta \hat{A}_{\Phi}\right]=\Delta \hat{B}_{\Phi}^{\dagger} \Delta \hat{A}_{\Phi}-\Delta \hat{A}_{\Phi}^{\dagger} \Delta \hat{B}_{\Phi},} \\
& \left\{\Delta \hat{B}_{\Phi}^{\dagger}, \Delta \hat{A}_{\Phi}\right\}=\Delta \hat{B}_{\Phi}^{\dagger} \Delta \hat{A}_{\Phi}+\Delta \hat{A}_{\Phi}^{\dagger} \Delta \hat{B}_{\Phi} .
\end{aligned}
$$

Using the definition of the difference operator given in Eq. (13) and the weak value difference as defined in Eq. (18) one finds with some algebra that respectively

$$
\begin{aligned}
& \frac{\left\langle\Psi_{t}\left|\left[\Delta \hat{B}_{\Phi}^{\dagger}, \Delta \hat{A}_{\Phi}\right]\right| \Psi_{t}\right\rangle}{\left|\left\langle\Psi_{t} \mid \Phi\right\rangle\right|^{2}}=\Delta B_{w}^{*}\left(\Phi ; \Psi_{t}\right) \Delta A_{w}\left(\Phi ; \Psi_{t}\right)-\Delta A_{w}^{*}\left(\Phi ; \Psi_{t}\right) \Delta B_{w}\left(\Phi ; \Psi_{t}\right), \\
& \frac{\left\langle\Psi_{t}\left|\left\{\Delta \hat{B}_{\Phi}^{\dagger}, \Delta \hat{A}_{\Phi}\right\}\right| \Psi_{t}\right\rangle}{\left|\left\langle\Psi_{t} \mid \Phi\right\rangle\right|^{2}}=\Delta B_{w}^{*}\left(\Phi ; \Psi_{t}\right) \Delta A_{w}\left(\Phi ; \Psi_{t}\right)+\Delta A_{w}^{*}\left(\Phi ; \Psi_{t}\right) \Delta B_{w}\left(\Phi ; \Psi_{t}\right) .
\end{aligned}
$$

Time averaging these results implies

$$
\begin{gathered}
\int_{0}^{\infty} d t \frac{\left|\left\langle\Psi_{t} \mid \Phi\right\rangle\right|^{2}}{N(\Phi ; \Psi)}\left[\frac{\left\langle\Psi_{t}\left|\left[\Delta \hat{B}_{\Phi}^{\dagger}, \Delta \hat{A}_{\Phi}\right]\right| \Psi_{t}\right\rangle}{\left|\left\langle\Psi_{t} \mid \Phi\right\rangle\right|^{2}}\right]=2 i \operatorname{Im}\left[\left\langle B_{w}^{*}\left(\Phi ; \Psi_{t}\right) A_{w}\left(\Phi ; \Psi_{t}\right)\right\rangle-\left\langle B_{w}^{*}\left(\Phi ; \Psi_{t}\right)\right\rangle\left\langle A_{w}\left(\Phi ; \Psi_{t}\right)\right\rangle\right] \\
\int_{0}^{\infty} d t \frac{\left|\left\langle\Psi_{t} \mid \Phi\right\rangle\right|^{2}}{N(\Phi ; \Psi)}\left[\frac{\left\langle\Psi_{t}\left|\left\{\Delta \hat{B}_{\Phi}^{\dagger}, \Delta \hat{A}_{\Phi}\right\}\right| \Psi_{t}\right\rangle}{\left|\left\langle\Psi_{t} \mid \Phi\right\rangle\right|^{2}}\right]=2 \operatorname{Re}\left[\left\langle B_{w}^{*}\left(\Phi ; \Psi_{t}\right) A_{w}\left(\Phi ; \Psi_{t}\right)\right\rangle-\left\langle B_{w}^{*}\left(\Phi ; \Psi_{t}\right)\right\rangle\left\langle A_{w}\left(\Phi ; \Psi_{t}\right)\right\rangle\right] .
\end{gathered}
$$

Therefore,

$$
\begin{aligned}
& \frac{1}{4}\left(\left[\int_{0}^{\infty} d t \frac{\left\langle\Psi_{t}\left|\left\{\Delta \hat{B}_{\Phi}^{\dagger}, \Delta \hat{A}_{\Phi}\right\}\right| \Psi_{t}\right\rangle}{N(\Phi ; \Psi)}\right]^{2}-\left[\int_{0}^{\infty} d t \frac{\left\langle\Psi_{t}\left|\left[\Delta \hat{B}_{\Phi}^{\dagger}, \Delta \hat{A}_{\Phi}\right]\right| \Psi_{t}\right\rangle}{N(\Phi ; \Psi)}\right]^{2}\right) \\
& \quad=\left(\operatorname{Re}\left[\left\langle B_{w}^{*}\left(\Phi ; \Psi_{t}\right) A_{w}\left(\Phi ; \Psi_{t}\right)\right\rangle-\left\langle B_{w}^{*}\left(\Phi ; \Psi_{t}\right)\right\rangle\left\langle A_{w}\left(\Phi ; \Psi_{t}\right)\right\rangle\right]\right)^{2}+\left(\operatorname{Im}\left[\left\langle B_{w}^{*}\left(\Phi ; \Psi_{t}\right) A_{w}\left(\Phi ; \Psi_{t}\right)\right\rangle-\left\langle B_{w}^{*}\left(\Phi ; \Psi_{t}\right)\right\rangle\left\langle A_{w}\left(\Phi ; \Psi_{t}\right)\right\rangle\right]\right)^{2}
\end{aligned}
$$

and this leads directly to Eq. (17).

[1] H. P. Robertson, Phys. Rev. 34, 163 (1929).

[2] E. Schrödinger, Phys.-math. Kl. 14, 296 (1930).

[3] M. Ozawa, Phys. Rev. A 67, 042105 (2003).

[4] Y. Aharonov, D. Z. Albert, and L. Vaidman, Phys. Rev. Lett. 60, 1351 (1988).

[5] H. M. Wiseman, New J. Phys. 9, 165 (2007).

[6] A. G. Kofman, S. Ashhab, and F. Nori, Phys. Rep. 520, 43 (2012).

[7] R. Flack and B. J. Hiley, J. Phys. Conf. Ser. 504, 012016 (2014).

[8] S. Kocsis, B. Braverman, S. Ravets, M. J. Stevens, R. P. Mirin, L. K. Shalm, and M. A. Steinberg, Science 332, 1170 (2011).

[9] J. S. Lundeen, B. Sutherland, A. Patel, C. Stewart, and C. Bamber, Nature (London) 474, 188 (2011).

[10] B. Tamir and E. Cohen, Quanta 2, 7 (2013).
[11] J. Dressel, M. Malik, F. M. Miatto, A. N. Jordan, and R. W. Boyd, Rev. Mod. Phys. 86, 307 (2014).

[12] A. P. Lund and H. M. Wiseman, New J. Phys. 12, 093011 (2010).

[13] L. A. Rozema, A. Darabi, D. H. Mahler, A. Hayat, Y. Soudagar, and A. M. Steinberg, Phys. Rev. Lett. 109, 100404 (2012).

[14] J. Petersen and E. Pollak, J. Phys. Chem. Lett. 8, 4017 (2017).

[15] E. Pollak and S. Miret-Artés, New. J. Phys. 20, 073016 (2018).

[16] Y. Aharonov and D. Bohm, Phys. Rev. 122, 1649 (1961).

[17] P. Busch, Lect. Notes Phys. 734, 73 (2008).

[18] T. Denkmayr, H. Geppert, H. Lemmel, M. Waegell, J. Dressel, Y. Hasegawa, and S. Sponar, Phys. Rev. Lett. 118, 010402 (2017). 
[19] T. Denkmayr, J. Dressel, H. Geppert-Kleinrath, Y. Hasegawa, and S. Sponar, Phys. B: Cond. Matter 551, 339 (2018).

[20] E. Cohen and E. Pollak, Phys. Rev. A 98, 042112 (2018).

[21] C. Cohen-Tannoudji, B. Diu, and F. Laloe, Quantum Mechanics (Wiley and Sons, New York, 1977), Vol. 1, pp. 286-287.
[22] E. Benítez Rodríguez, L. M. Arévalo Aguilar, and E. Piceno Martínez, Eur. J. Phys. 38, 069501 (2017).

[23] S. Sponar, T. Denkmayr, H. Geppert, H. Lemmel, A. Matzkin, J. Tollaksen, and Y. Hasegawa, Phys. Rev. A 92, 062121 (2015). 